\title{
(2) OPEN ACCESS \\ Reducing door-to-reperfusion time in acute stroke endovascular therapy using magnetic resonance imaging as a screening modality
}

\author{
Yuki Sakamoto @ ( , Kentaro Suzuki, Arata Abe, Junya Aoki, Takuya Kanamaru, \\ Yohei Takayama, Takehiro Katano, Akihito Kutsuna, Satoshi Suda, Yasuhiro Nishiyama, \\ Chikako Nito, Kazumi Kimura
}

\begin{abstract}
- Additional material is published online only. To view please visit the journal online (http://dx.doi.org/10.1136/ neurintsurg-2019-015625).
\end{abstract}

Neurology, Nippon Medical School, Tokyo, Japan

Correspondence to Dr Yuki Sakamoto, Department of Neurology, Nippon Medical School, Tokyo 113-8602, Japan; ysakamoto02@gmail.com

Received 12 November 2019 Revised 13 January 2020 Accepted 22 January 2020 Published Online First 12 February 2020

\begin{abstract}
Background The feasibility of performing MRI first for patients with suspected hyperacute stroke in real-world practice has not been fully examined. Moreover, most past studies of reducing door-to-reperfusion time (DRT) in endovascular treatment (EVT) were conducted using CT. The aim of this study was to evaluate the feasibility of an MRI-first policy and to examine the effects of a quality improvement (QI) process for reducing DRT using MRI.
\end{abstract} Methods From January 2013 to December 2018, consecutive patients with acute stroke who came to hospital directly and were treated with emergent EVT were prospectively enrolled into the present study. In principle, MRI was performed first for patients with suspected acute stroke. A step-by-step QI process for decreasing DRT was adopted during this period. Time metrics for EVT were compared between specific time periods.

Results A total of 180 patients (71 women; median age 76 years (range 69-64); National Institutes of Health Stroke Scale score 17 (range 10-23)) were included in the present study. More patients in the late phase were managed with the MRI-first policy $(p<0.001)$. DRT (199 min in Phase 1, $135 \mathrm{~min}$ in Phase 2, $129 \mathrm{~min}$ in Phase 3, and $121 \mathrm{~min}$ in Phase $4, p<0.001$ ) was significantly reduced across the phases. The percentage of patients with DRT $<120$ min increased significantly across time periods $(p<0.001)$. Symptomatic intracerebral hemorrhage did not increase across phases $(p=0.575)$. Conclusion An MRI-first policy was feasible, and DRT decreased considerably with a step-by-step QI process. This process may be applicable to other hospitals.

\section{INTRODUCTION}

Time from symptom onset to reperfusion plays a critical role in managing ischemic stroke patients with emergent large vessel occlusion (ELVO), ${ }^{1-3}$ and urgent endovascular treatment (EVT) for reperfusion is recommended for most stroke patients with ELVO. ${ }^{4}$ Because the probability of a good functional outcome decreases as stroke onset to reperfusion time increases, ${ }^{13}$ guidelines or statements emphasize the importance of minimizing onset to reperfusion time. ${ }^{45}$ For in-hospital workflow, some time metrics have been proposed, such as door-to-imaging time (DIT), door-to-puncture time (DPT), and door-to-reperfusion time (DRT).
In the ideal stroke process timelines suggested by the Society of NeuroInterventional Surgery statement, DPT should be $<60 \mathrm{~min}$ and DRT $<90 \mathrm{~min}^{5}$ Various reports of reducing these time metrics using quality improvement (QI) processes have been published. $^{6-9}$

In most past studies trying to reduce these time metrics, CT and/or CT angiography (CTA) was performed as a screening modality for hyperacute ischemic stroke. CT has some advantages, such as requiring a short time and availability at any time in almost all hospitals with an emergency department (ED). However, there is controversy about patient selection by CT/CTA or MRI. MRI-based patient selection has a potential to better screen patients for candidates for EVT in terms of their likelihood to benefit from immediate reperfusion therapy. ${ }^{10}$ Diffusion-weighted imaging (DWI) depicts the extent of the ischemic core lesion well, ${ }^{11}$ and easily shows patients with a large ischemic core. Moreover, MR angiography (MRA) can show the site of arterial occlusion without contrast medium injection, and such information may be beneficial for considering subsequent EVT. However, few institutions perform MRI as an initial examination for patients with suspected acute stroke ${ }^{12}$ because it is much more time-consuming than $\mathrm{CT}^{1314}$ and is probably not always available, and the feasibility of performing MRI first for patients with suspected acute stroke in real-world practice has not been examined sufficiently. ${ }^{12}$

The aim of the present study was to evaluate the feasibility of an MRI-first policy in conducting EVT and to examine the effect of a step-by-step QI process for decreasing DRT using MRI as the routine screening modality.

\section{METHODS}

The Nippon Medical School is a major general academic teaching hospital in central Tokyo. It is a tertiary general hospital and treats more than 10000 patients per year through the ED, including $>600$ stroke admissions annually, and CT and MRI are available at any time. ${ }^{12}$

From January 2013 to December 2018, consecutive patients with acute ischemic stroke who were treated with emergent EVT at admission were retrospectively recruited from the prospective registry. Patients treated with EVT after admission (eg, for 
symptom progression) or patients who were attempted to be treated with EVT but with no procedure (eg, effective recanalization on first angiogram after intravenous tissue plasminogen activator (t-PA)) were excluded. EVT for ELVO patients was conducted generally based on, but not limited to, our domestic guideline (briefly, EVT was strongly recommended for stroke patients within 6 hours from symptom onset, with internal carotid artery or middle cerebral artery horizontal segment occlusion, Alberta Stroke Program Early CT Score (ASPECTS) $\geq 6$, and National Institutes of Health Stroke Scale (NIHSS) score of $\geq 6$; EVT was also recommended for patients within 24 hours from onset, with ASPECTS $\geq 7$ and NIHSS $\geq 6$ ). ${ }^{15}$ For candidate patients outside the guideline, EVT was conducted based on clinical-DWI mismatch, and generally there were no limits about time window, ASPECTS, NIHSS score, or the site of arterial occlusion (distal and/or posterior circulation).

This study was approved by the institutional ethics committee. Written informed consent for enrollment into the study and data collection was obtained from all patients or their next of kin.

\section{Clinical characteristics of patients}

Clinical background characteristics including sex, age, vascular risk factors, and time of arrival at the ED (with office hours defined as Monday to Friday, 08:30-16:00 hours) were recorded on admission. Stroke severity was assessed using the NIHSS. The site of arterial occlusion was determined using the first angiogram. Time metrics related to hyperacute stroke management such as DIT, DPT, DRT, imaging-to-puncture time (IPT), and puncture-to-reperfusion time (PRT) were also recorded. Successful reperfusion was defined as a modified Thrombolysis in Cerebral Infarction score ${ }^{16}$ of $2 b$ or 3 . Concomitant t-PA treatment and symptomatic intracerebral hemorrhage $(\mathrm{sICH})$ were documented. For patients treated with intravenous t-PA, door-to-needle time was also recorded. sICH was defined as new parenchymal hemorrhage within 36 hours after EVT, corresponding to an increment in the NIHSS score of $\geq 4$ points. Stroke etiology was determined using the Trial of ORG 10172 in Acute Stroke Treatment (TOAST) criteria, ${ }^{17}$ and functional outcomes were classified by the modified Rankin scale (mRS) score ${ }^{18} 3$ months after stroke onset.

\section{Step-by-step QI process for decreasing DRT}

The step-by-step quality improvement process for decreasing DRT is shown in table 1.
Phase 1: January 2013 to February 2015

From January 2013, multimodal 1.5 T MRI (Echelon Oval, Hitachi Medical Systems, Tokyo, Japan) was performed first for patients suspected of having hyperacute stroke $(<4$ hours from onset at ED arrival) and who arrived at the hospital within office hours in principle; exceptions to the MRI-first policy were permitted at the attending neurologist's discretion if the patient had contraindications to MRI, the general condition of the patient was poor, or MRI was unavailable due to its use for other patients. The patients were screened by a vascular neurology fellow or attending neurologist both during and outside office hours (throughout the following phases). DWI (TR/TE 6000/65 ms; b-values 0 and $1000 \mathrm{~s} / \mathrm{mm}^{2}$; field of view $24 \mathrm{~cm}$; acquisition matrix $128 \times 128$; and slice thickness $4.5 \mathrm{~mm}$, with a $2.5 \mathrm{~mm}$ intersection gap, takes $55 \mathrm{~s}$ ), time-of-flight MRA (330s), fluidattenuated inversion recovery (FLAIR, TR/TE 10000/120 ms; TI $2500 \mathrm{~ms}$; field of view $24 \mathrm{~cm}$; acquisition matrix $288 \times 224$; and slice thickness $4.5 \mathrm{~mm}$, with a $2.5 \mathrm{~mm}$ intersection gap, $161 \mathrm{~s}$ ), T1-weighted imaging (TR/TE 540/12 ms, $140 \mathrm{~s}$ ), T2-weighted imaging (TR/TE 4200/117.52 ms, $173 \mathrm{~s}$ ), and T2*-weighted imaging (TR/TE 480/18 ms; field of view $24 \mathrm{~cm}$; acquisition matrix $256 \times 204$; and slice thickness $4.5 \mathrm{~mm}$, with a $2.5 \mathrm{~mm}$ intersection gap, 136s) were performed routinely in this phase. For patients screened with CT, additional imaging (CTA or MRA) could be performed, mainly to assess large vessel occlusion. Our stroke unit opened and pre-notification by the emergency medical service (EMS) for suspected acute stroke patients was started during this phase.

\section{Phase 2: March 2015 to March 2016}

The stroke management system was re-evaluated by a working group from neurology, emergency, radiology, and nursing. MRI was performed first for patients suspected of having acute stroke regardless of onset-to-door time and time of arrival (office hours or not). Routine MRI sequences were limited to DWI, MRA (faster protocol (200s) was adopted), FLAIR, and T2*-weighted imaging, and rapid examination point-of-care tools to measure complete blood cell count (Celltac ES, NIHON KOHDEN Corporation, Tokyo, Japan), serum glucose, creatinine (ABL800 FLEX, Radiometer, Copenhagen, Denmark), and partial thrombin time-international normalized ratio (CoaguCheck XS, Roche Diagnostics, Rotkreuz, Switzerland) were introduced. In this phase, patients with ELVO having an indication for t-PA were transferred from the imaging room to the stroke unit and started on intravenous t-PA, and then delivered to the angiosuite.

Table 1 Step-by-step quality improvement

\begin{tabular}{|c|c|c|c|c|}
\hline & Phase 1 & Phase 2 & Phase 3 & Phase 4 \\
\hline & January 2013-February 2015 & March 2015-March 2016 & April 2016-August 2017 & $\begin{array}{l}\text { September 2017-December } \\
2018\end{array}$ \\
\hline MRI-first policy & $<4$ hours from onset, in office hours & Any patient, any time & Any patient, any time & Any patient, any time \\
\hline MRI sequence & DWI, MRA, FLAIR, T1, T2, T2* & DWI, MRA, FLAIR, T2* & DWI, MRA, FLAIR, T2* & DWI, MRA, FLAIR, T2* \\
\hline MRI acquisition time & $16 \min 30 s$ & $9 \min 12 \mathrm{~s}$ & $9 \min 12 \mathrm{~s}$ & $9 \min 12 \mathrm{~s}$ \\
\hline Rapid point-of-care examination tool & None & Implemented & Implemented & Implemented \\
\hline Concomitant t-PA & $\mathrm{t}-\mathrm{PA}$ in $\mathrm{SU} \rightarrow$ Angiosuite & $\mathrm{t}-\mathrm{PA}$ in $\mathrm{SU} \rightarrow$ Angiosuite & t-PA in Angiosuite & t-PA in Angiosuite \\
\hline Multidisciplinary meeting & None & None & Held once a month & Held once a month \\
\hline Stent retrievers & None & None & None & Implemented \\
\hline
\end{tabular}

DWI, diffusion-weighted imaging; FLAIR, fluid-attenuated inversion recovery; MRA, magnetic resonance angiography; MRI, magnetic resonance imaging; SU, stroke unit; t-PA, tissue plasminogen activator. 
Table 2 Clinical characteristics of the included patients

\begin{tabular}{|c|c|c|c|c|c|}
\hline & $\begin{array}{l}\text { Phase } 1 \\
26 \text { months }\end{array}$ & $\begin{array}{l}\text { Phase } 2 \\
13 \text { months }\end{array}$ & $\begin{array}{l}\text { Phase } 3 \\
17 \text { months }\end{array}$ & $\begin{array}{l}\text { Phase } 4 \\
16 \text { months }\end{array}$ & \\
\hline Variable & $n=26$ & $n=30$ & $\mathrm{n}=68$ & $n=56$ & $P$ value \\
\hline Female sex, $\mathrm{n}(\%)$ & $11(42)$ & $12(40)$ & $25(37)$ & $23(41)$ & 0.948 \\
\hline Age, years, median (IQR) & $78(68-85)$ & $76(67-83)$ & $76(69-84)$ & $75(69-82)$ & 0.856 \\
\hline EMS pre-notification, $\mathrm{n}(\%)$ & $18(69)$ & $27(90)$ & $57(84)$ & $43(77)$ & 0.186 \\
\hline Arrival in daytime on weekdays, $\mathrm{n}(\%)$ & $13(50)$ & $10(33)$ & $29(43)$ & $16(29)$ & 0.203 \\
\hline NIHSS score on admission, median (IQR) & $22(16-29)$ & $17(11-22)$ & $17(8-20)$ & $15(10-23)$ & 0.016 \\
\hline Onset-to-reperfusion time, min, median (IQR) & $310(230-807)$ & $225(166-332)$ & $218(156-383)$ & $235(164-524)$ & 0.032 \\
\hline Onset-to-door time, min, median (IQR) & $95(40-482)$ & $82(53-282)$ & $80(48-253)$ & $106(49-419)$ & 0.673 \\
\hline Door-to-reperfusion time, min, median (IQR) & $199(172-244)$ & $135(108-168)$ & $129(99-165)$ & $121(94-157)$ & $<0.001$ \\
\hline Door-to-imaging time, min, median (IQR) & $31(22-39)$ & $23(20-28)$ & $27(21-33)$ & $23(19-32)$ & 0.062 \\
\hline Imaging-to-puncture time, min, median (IQR) & $95(84-119)$ & $57(42-79)$ & $49(38-71)$ & $50(38-79)$ & $<0.001$ \\
\hline Puncture-to-reperfusion time, min, median (IQR) & $68(44-107)$ & $52(28-72)$ & $41(27-70)$ & $38(22-59)$ & 0.003 \\
\hline Door-to-reperfusion time <90 min, $\mathrm{n}(\%)$ & $0(0)$ & $2(7)$ & $11(16)$ & $10(18)$ & 0.078 \\
\hline Door-to-reperfusion time $<120 \mathrm{~min}, \mathrm{n}(\%)$ & $1(4)$ & $11(37)$ & $33(49)$ & $28(50)$ & $<0.001$ \\
\hline Using stent retrievers, $\mathrm{n}(\%)$ & $1(4)$ & $5(17)$ & $19(28)$ & $28(50)$ & $<0.001$ \\
\hline Successful reperfusion* ${ }^{*} \mathrm{n}(\%)$ & $20(77)$ & $25(83)$ & $57(84)$ & $44(79)$ & 0.812 \\
\hline MRI-first, n (\%) & $2(8)$ & $16(53)$ & $52(77)$ & $46(82)$ & $<0.001$ \\
\hline Major artery occlusiont on admission, $\mathrm{n}(\%)$ & $24(92)$ & $20(67)$ & $45(66)$ & $27(48)$ & 0.002 \\
\hline Concomitant t-PA treatment, $\mathrm{n}(\%)$ & $12(46)$ & $17(57)$ & $22(32)$ & $19(34)$ & 0.095 \\
\hline Door-to-needle time, min, median (IQR) $\ddagger$ & $80(74-119)$ & $66(53-76)$ & $63(57-86)$ & $60(55-76)$ & 0.069 \\
\hline Symptomatic intracerebral hemorrhage, n (\%) & $3(11.5)$ & $2(6.7)$ & $4(5.9)$ & $2(3.6)$ & 0.575 \\
\hline Cardioembolic stroke on TOAST criteria, $\mathrm{n}(\%)$ & $24(92)$ & $20(67)$ & $44(65)$ & $33(59)$ & 0.025 \\
\hline mRS score $0-2$ at 3 months after stroke, $\mathrm{n}(\%)$ & $12(46)$ & $16(53)$ & $29(43)$ & $23(41)$ & 0.720 \\
\hline
\end{tabular}

Phase 1: From January 2013 to February 2015.

Phase 2: From March 2015 to March 2016.

Phase 3: From April 2016 to August 2017.

Phase 4: From September 2017 to December 2018.

*Defined as Thrombolysis in Cerebral Infarction score $2 \mathrm{~b}$ or 3.

tInternal carotid artery, middle cerebral artery horizontal segment, and basilar artery occlusion.

\#For only patients treated with intravenous tissue-plasminogen activator.

EMS, emergency medical service; MRI, magnetic resonance imaging; mRS, modified Rankin Scale; NIHSS, National Institutes of Health Stroke Scale; TOAST, Trial of ORG 10172 in

Acute Stroke Treatment; t-PA, tissue plasminogen activator.

The details of the QI processes in Phases 1 and 2 have also been described elsewhere. ${ }^{12}$

\section{Phase 3: April 2016 to August 2017}

From April 2016, the hyperacute stroke management system had further improved for reducing DPT. Patients with ELVO were directly transferred to the angiosuite from the imaging room, and t-PA was started wherever. A pre-hospital scale that could predict ELVO was introduced with cooperation of the EMS during this phase. ${ }^{19}$ Multidisciplinary staff meetings were held to review past cases or to familiarize them with the stroke management system for suspected ELVO patients through role play.

\section{Phase 4: September 2017 to December 2018}

Stent retrievers and large (0.068 inch) inner lumen aspiration catheters were introduced as on-label in our institution from September 2017.

\section{Statistical analyses}

First, the clinical characteristics of the patients including the proportion of patients who received MRI first, time metrics, and the ratio of cases with DRT $<90$ or $<120 \mathrm{~min}$ of the included patients were compared among the phases. For this purpose, the time of the final angiogram was set as the time of reperfusion when reperfusion was not achieved. Because patients with ELVO stroke transferred from a primary stroke center (confirmed ELVO diagnosis, always with pre-notification, and skip head imaging and laboratory tests in our hospital) or patients with in-hospital onset (essentially onset-to-door time (ODT) is zero and never pre-notified) have distinct characteristics from patients who come directly to our hospital ${ }^{20}$ (see online supplementary table 1), only cases who came directly to our hospital were assessed in the present study. Univariate analyses were performed using the $\chi^{2}$ test, Fisher's exact test, the Kruskal-Wallis test, or the Mann-Whitney U test, as appropriate. The data are presented as median (IQR) or numbers (\%). Second, differences in time metrics were evaluated according to several subgroups: arrival timing, EMS pre-notification, and first imaging modality. Finally, factors affecting DRT in Phases 3 and 4 were analyzed using multiple linear regression analysis with a backward stepwise selection procedure. Sex, age, risk factors, etiology, EMS prenotification, arrival timing, ODT, severity, first imaging modality, major artery occlusion, and t-PA use were included in the model. All statistical analyses were performed using PASW for Windows 
All Patients $(\mathrm{n}=180, \mathrm{p}<0.001)$

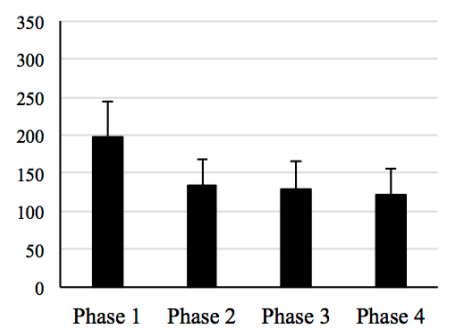

Daytime $(\mathrm{n}=68, \mathrm{p}=0.039)$

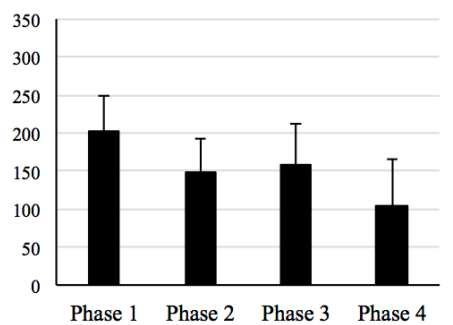

Prenotice $(n=145, p<0.001)$

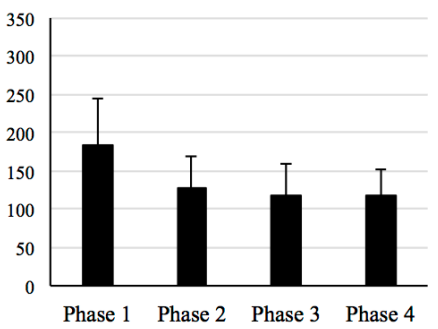

MRI-First $(n=116, p=0.208)$

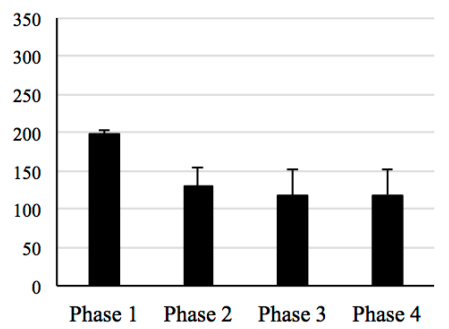

Night $(n=112, p<0.001)$

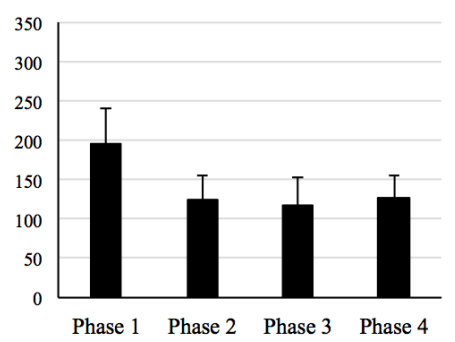

Non-Prenotice $(n=35, p=0.415)$

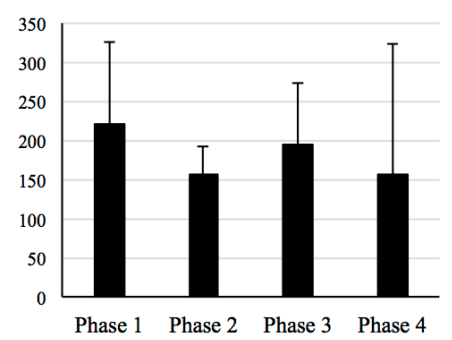

CT-First $(n=64, p=0.025)$

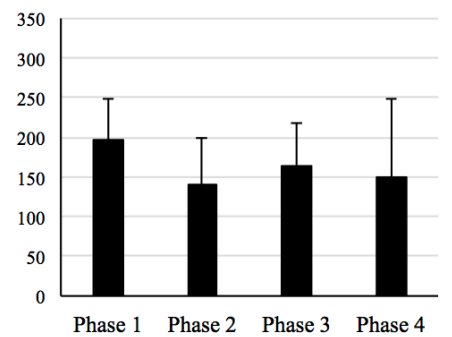

Figure 1 Door-to-reperfusion time (black boxes) in phases 1-4 according to various subgroups. Boxes and bars show median values and IQR.

version 17.0 software (SPSS, Chicago, Illinois, USA). Results were considered significant at $\mathrm{p}<0.05$.

\section{RESULTS}

From January 2013 through December 2018, 2344 patients with acute ( $<7$ days from onset) ischemic stroke were admitted to our stroke center: 706 in Phase 1, 442 in Phase 2, 638 in Phase 3, and 558 in Phase 4. During this period, a total of 355 EVTs (41 (1.6/ month) in Phase 1, 48 (3.7/month) in Phase 2, 117 (6.9/month) in Phase 3, and 149 (9.3/month) in Phase 4) were conducted and, of these 355 EVTs, 326 were for ELVO. The rate of patients treated with EVT of those with acute stroke was significantly different among the phases $(5.8 \%$ in Phase $1,10.9 \%$ in Phase 2, 18.3\% in Phase 3, and $26.7 \%$ in Phase $4, \mathrm{p}<0.001)$. The online supplementary table shows the clinical background characteristics of the patients with ELVO who received EVT. The prevalence of risk factors, premorbid mRS score $(\mathrm{p}=0.002)$, time metrics of EVT, the proportion of skip head imaging $(\mathrm{p}<0.001)$, major artery occlusion $(p=0.016)$, and concomitant $t-P A$ therapy $(p=0.006)$ were different among patients with ELVO who arrived at our hospital directly, those transferred from a primary stroke center, or those who had in-hospital onset. Therefore, only patients with ELVO who came to our hospital directly ( $\mathrm{n}=180$ : 71 women; median age 76 (69-84) years; NIHSS score 17) were included in the present study.

Table 2 shows the clinical background characteristics of the included patients. Sex, age, the rates of EMS pre-notification and of patients who arrived at the ED during office hours, and ODT did not differ between the four phases. In the late phase, fewer patients had major artery occlusion $(p=0.002)$ and cardioembolic stroke $(p=0.025)$. More patients were managed with the MRI-first policy in the late phase $(p<0.001)$. The main reason for not following the MRI-first policy, especially in the early phase, was the unavailability of MRI due to examining other patients. There was no adverse event due to an MRI-first policy, such as a metal absorption-associated accident. DRT was significantly decreased across the phases $(199 \mathrm{~min}$ in Phase 1, $135 \mathrm{~min}$ in Phase 2, $129 \mathrm{~min}$ in Phase 3, and $121 \mathrm{~min}$ in Phase $4, p<0.001$, figure 1). Both IPT $(p<0.001)$ and PRT $(p=0.003)$ decreased across the phases: IPT and PRT in Phase 4 were roughly half of those in Phase 1 . The percentage of patients with DRT $<90$ min or DRT $<120$ min increased across the time periods, especially DRT $<120 \mathrm{~min}$ (4\% in Phase 1, 37\% in Phase 2, 49\% in Phase 3, and 50\% in Phase 4, p<0.001). sICH did not increase across the phases $(p=0.575)$. These results in time metrics did not change significantly even when only patients with successful reperfusion $(n=146)$ were evaluated.

Figure 1 shows DRT in several subgroups. DRT decreased irrespective of arrival time or first imaging modality. However, in patients with ELVO without pre-notification by EMS, DRT did not change across the phases. DRT in patients screened using MRI seemed to decrease, but the reduction was not statistically significant $(p=0.208)$, probably due to the extremely small number of MRI-screened patients in Phase 1 (table 2). On multiple linear regression analysis, EMS pre-notification (standardized coefficient $-0.409, \mathrm{p}<0.001$ ), ODT (standardized coefficient 0.256 per $60 \mathrm{~min}$ increase, $\mathrm{p}=0.001$ ), and cardioembolism (standardized coefficient $-0.175, \mathrm{p}=0.026$ ) were the independent predictors of DRT in Phases 3 and 4 (table 3).

Table 3 Multiple linear regression analysis for independent predictors of the door-to-reperfusion time in phases 3 and 4

\begin{tabular}{llrrr}
\hline Variable & $\begin{array}{l}\text { Unstandardized coefficient } \\
(95 \% \mathrm{Cl})\end{array}$ & \multicolumn{1}{l}{ SE } & Standardized coefficient & P value \\
\hline EMS pre-notification & $-98.7(-136.1$ to -61.3$)$ & 18.9 & -0.409 & $<0.001$ \\
Onset-to-door time (per 60 min increment) & $4.7(1.9$ to 7.6$)$ & 1.4 & 0.256 & 0.001 \\
Cardioembolism & $-34.2(-64.3$ to -4.1$)$ & 15.2 & -0.175 & 0.026 \\
\hline
\end{tabular}

The variables identified by the backward selection procedure are listed.

EMS, emergency medical service. 
There were no variables with a variance inflation factor (indicator of multicollinearity) $>2$.

\section{DISCUSSION}

The results of the present study show that an MRI-first policy for patients suspected of having acute stroke is feasible. In addition, a step-by-step QI process decreased DRT. Although the QI process effectively reduced DRT in most patients with ELVO, there seemed to be room for improvement for patients without EMS pre-notification.

The feasibility of an MRI-first policy for patients with suspected acute stroke and ELVO in a tertiary general academic teaching hospital is not well known, probably because few institutions perform MRI as an initial examination for patients with suspected acute stroke. ${ }^{21}$ An MRI-first policy was feasible; more than $80 \%$ of the included patients were screened with MRI, and half of those were treated with EVT $<120$ min after arrival in Phase 4 of the present study. Moreover, time metrics such as DPT were comparable to those of a real-world multicenter registry. ${ }^{22}$ An MRI-first policy could be adopted without an increment in serious adverse events or time metrics about EVT.

MRI takes a slightly longer time than $\mathrm{CT},{ }^{23}$ but it clearly depicts the extent of the ischemic lesion on DWI. DWI can delineate small and/or brainstem lesions. In this respect MRI is superior to CT, especially in hospitals where various levels of physicians may manage patients with suspected hyperacute ischemic stroke, as in our institution. Moreover, MRA can show the site of arterial occlusion without contrast medium injection, and this is a huge advantage of MRI, particularly in Japan because written consent is generally needed before using contrast medium in Japan. For patients with hyperacute stroke, DWI hyperintensity brings an accurate and clear diagnosis of ischemic stroke and plain radiological indications and contraindications for EVT. Indeed, the rate of sICH was gradually reduced from $11.5 \%$ in Phase 1 to $3.6 \%$ in Phase 4, which may have been in part because the ratio of patients screened by MRI increased (8\% in Phase 1 through 82\% in Phase 4).

DRT was decreased by 78 min with a step-by-step QI approach. This result was in line with past studies which showed that a QI process could shorten DRT using CT as a screening modality, ${ }^{67}$ but there are few reports on reducing DRT using MRI as a screening modality. Moreover, the DRT reduction was recorded in most subgroups, including both during and outside office hours. The time metrics of EVT were longer outside office hours than during office hours in past reports, ${ }^{24} 25$ and a systematic protocol reduced DRT but had no effect outside office hours. ${ }^{86}$ Screening of patients by a vascular neurology fellow or attending neurologist both during and outside office hours, along with a step-by-step approach, may facilitate shortening DRT in various scenarios in large hospitals.

EMS pre-notification was an independent predictor for DRT, and DRT in patients without EMS pre-notification did not decrease over time. The reason for the lack of improvement is clear, as such patients come to hospital by themselves or with EMS, are examined first by doctors in the ED or physicians from a department other than neurology, and then neurologists are called. DRT in patients with ELVO without pre-notification tended to be longest among various subgroups (figure 1); therefore, education and explanation of symptoms suggestive of $\mathrm{ELVO}^{19}$ or rapid response to possible ELVO cases by EMS or all staff in the ED is critically and urgently needed.

This study has some limitations that need to be addressed. First, the DRT in patients screened using MRI did not change during the study period, and the ideal DRT could not be achieved when MRI was used as a first-line screening modality, at least with the protocol used in the present study. Although the time metrics in the present study were comparable to a real-world registry, ${ }^{22}$ our workflow may need further improvements. Second, the present study is a retrospective study from a prospective real-world registry, and all the patients who arrived directly at the hospital and received emergent EVT were recruited from the registry. Patients with acute stroke outside the definite evidence (late time window, distal artery occlusion, or posterior circulation stroke) were also included, especially in the late phase. In addition, the proportion of patients treated with stent retrievers was not high (50\% even in Phase 4), and modern automated post-processing software (such as RAPID) is not adopted in our institution. DRT between Phases 3 and 4 or functional outcome 3 months after stroke did not improve in the present study, probably due to such limitations. Third, the number of patients included in the present study was relatively small. Some subgroup analyses (especially patients screened with MRI) may be statistically underpowered due to the small number of subgroup patients. Fourth, the hardware had also changed during the study period (the stroke unit was opened during Phase 1). The QI process included an intervention for stroke care, the MRI acquisition protocol, and patient logistics. In addition, the number of EVT procedures increased steeply during the study period (from $1.6 /$ month to 9.3 (month). DRT reduction in the present study should be achieved by these changes, ${ }^{27}$ but the present study could not analyze these effects separately. Fifth, the present study was conducted in a single tertiary general academic teaching hospital, and its results may not be generalizable to all hospitals.

\section{CONCLUSION}

An MRI-first policy was feasible in a tertiary general academic teaching hospital. DRT was decreased substantially in most subgroups with a step-by-step QI process, probably along with an increment in the number of EVT procedures per month. Notification regarding patients with suspected ELVO both in the pre-hospital and in-hospital stage is needed for further DRT reduction and patient benefit.

Acknowledgements The authors would like to express their deepest gratitude to all members of the stroke unit and radiology and emergency departments. They would also like to thank Chie Ushiki for her assistance with database registration.

Contributors YS framed the study concept, collected data, wrote and revised this manuscript. KS, AA, JA, TK, YT, TK, and AK performed the endovascular procedure, collected data, and offered scientific advice. SS, YN, and CN offered scientific advice for the concept, data collection, and interpreting data. KK offered scientific advice for the concept, data collection, interpreting data, and revision of the manuscript.

Funding This work was partly supported by JSPS KAKENHI Grant Number JP19K23985.

Competing interests None declared.

Patient consent for publication Not required.

Provenance and peer review Not commissioned; externally peer reviewed.

Data availability statement Data are available upon reasonable request.

Open access This is an open access article distributed in accordance with the Creative Commons Attribution Non Commercial (CC BY-NC 4.0) license, which permits others to distribute, remix, adapt, build upon this work non-commercially, and license their derivative works on different terms, provided the original work is properly cited, appropriate credit is given, any changes made indicated, and the use is non-commercial. See: http://creativecommons.org/licenses/by-nc/4.0/.

\section{ORCID iD}

Yuki Sakamoto http://orcid.org/0000-0001-8353-1787

\section{REFERENCES}

1 Fransen PSS, Berkhemer OA, Lingsma HF, et al. Time to reperfusion and treatment effect for acute ischemic stroke: a randomized clinical trial. JAMA Neurol 2016;73:190-6.

2 Goyal M, Jadhav AP, Bonafe A, et al. Analysis of workflow and time to treatment and the effects on outcome in endovascular treatment of acute ischemic stroke: results from the SWIFT PRIME randomized controlled trial. Radiology 2016;279:888-97. 
3 Saver JL, Goyal M, van der Lugt A, et al. Time to treatment with endovascular thrombectomy and outcomes from ischemic stroke: a meta-analysis. JAMA 2016;316:1279-88.

4 Powers WJ, Rabinstein AA, Ackerson T, et al. Guidelines for the early management of patients with acute ischemic stroke: a guideline for healthcare professionals from the American Heart Association/American Stroke Association. Stroke 2018;2018:e46-110

5 McTaggart RA, Ansari SA, Goyal M, et al. Initial hospital management of patients with emergent large vessel occlusion (ELVO): report of the standards and guidelines Committee of the Society of Neurolnterventional Surgery. J Neurointerv Surg 2017:9:316-23

6 Rai AT, Smith MS, Boo S, et al. The 'pit-crew' model for improving door-to-needle times in endovascular stroke therapy: a Six-Sigma project. J Neurointerv Surg 2016;8:447-52.

7 Aghaebrahim A, Streib C, Rangaraju S, et al. Streamlining door to recanalization processes in endovascular stroke therapy. J Neurointerv Surg 2017;9:340-5.

8 Mehta BP, Leslie-Mazwi TM, Chandra RV, et al. Reducing door-to-puncture times for intra-arterial stroke therapy: a pilot quality improvement project. J Am Heart Assoc 2014:3:e000963.

9 Schregel K, Behme D, Tsogkas I, et al. Effects of workflow optimization in endovascularly treated stroke patients - a pre-post effectiveness study. PLoS One 2016;11:e0169192.

10 Leslie-Mazwi TM, Hirsch JA, Falcone GJ, et al. Endovascular stroke treatment outcomes after patient selection based on magnetic resonance imaging and clinical criteria. JAMA Neurol 2016;73:43-9.

11 Minematsu K, Li L, Fisher M, et al. Diffusion-weighted magnetic resonance imaging: rapid and quantitative detection of focal brain ischemia. Neurology 1992;42:235-40

12 Sakamoto Y, Tanabe M, Masuda K, et al. Feasibility of using magnetic resonance imaging as a screening tool for acute stroke thrombolysis. J Neuro/ Sci 2016;368:168-72

13 Sheth KN, Terry JB, Nogueira RG, et al. Advanced modality imaging evaluation in acute ischemic stroke may lead to delayed endovascular reperfusion therapy without improvement in clinical outcomes. J Neurointerv Surg 2013;5 Suppl 1:i62-5.

14 Menon BK, Almekhlafi MA, Pereira VM, et al. Optimal workflow and processbased performance measures for endovascular therapy in acute ischemic stroke: analysis of the Solitaire FR thrombectomy for acute revascularization study. Stroke 2014;45:2024-9.
15 The Japan Stroke Society, The Japan Neurosurgical Society, and The Japanese Society for Neuroendovascular Therapy. Guidelines for proper use of percutaneous transluminal mechanical cerebral thrombectomy devices 3rd edition. Nosochu 2018:40:285-309.

16 Zaidat 00, Yoo AJ, Khatri P, et al. Recommendations on angiographic revascularization grading standards for acute ischemic stroke: a consensus statement. Stroke 2013:44:2650-63.

17 Adams HP, Bendixen BH, Kappelle LJ, et al. Classification of subtype of acute ischemic stroke. Definitions for use in a multicenter clinical trial. TOAST. Trial of ORG 10172 in Acute Stroke Treatment. Stroke 1993:24:35-41.

18 van Swieten JC, Koudstaal PJ, Visser MC, et al. Interobserver agreement for the assessment of handicap in stroke patients. Stroke 1988;19:604-7.

19 Suzuki K, Nakajima N, Kunimoto K, et al. Emergent large vessel occlusion screen is an ideal prehospital scale to avoid missing endovascular therapy in acute stroke. Stroke 2018:49:2096-101.

20 Aoki J, Suzuki K, Kanamaru T, et al. [To optimize the initial assessment for stroke patients transferred from general hospital may improve the clinical outcome after endovascular thrombectomy]. Rinsho Shinkeigaku 2018;58:471-8.

21 Hjort N, Butcher K, Davis SM, et al. Magnetic resonance imaging criteria for thrombolysis in acute cerebral infarct. Stroke 2005;36:388-97.

22 Zaidat 00, Castonguay AC, Nogueira RG, et al. TREVO stent-retriever mechanical thrombectomy for acute ischemic stroke secondary to large vessel occlusion registry. $J$ Neurointerv Surg 2018;10:516-24.

23 Provost C, Soudant M, Legrand L, et al. Magnetic resonance imaging or computed tomography before treatment in acute ischemic stroke. Stroke 2019;50:659-64.

24 Almallouhi E, Al Kasab S, Harvey JB, et al. Impact of treatment time on the longterm outcome of stroke patients treated with mechanical thrombectomy. I Stroke Cerebrovasc Dis 2019:28:185-90.

25 Goyal M, Almekhlafi MA, Fan L, et al. Evaluation of interval times from onset to reperfusion in patients undergoing endovascular therapy in the Interventional Management of Stroke III trial. Circulation 2014;130:265-72.

26 van Heerden J, Yan B, Churilov L, et al. Picture-to-puncture time in acute stroke endovascular intervention: are we getting faster? J Neurointerv Surg $2015 ; 7: 564-8$

27 Gupta R, Horev A, Nguyen T, et al. Higher volume endovascular stroke centers have faster times to treatment, higher reperfusion rates and higher rates of good clinical outcomes. J Neurointerv Surg 2013:5:294-7. 